\title{
Transitioning CMS to Rucio Data Management
}

Eric Vaandering - for the CMS Rucio team - Presented by Nick Smith CHEP 2019 - Adelaide, Australia

4 November 2019

This document was prepared by CMS collaboration using the resources of the Fermi National Accelerator Laboratory (Fermilab), a U.S. Department of Energy, Office of Science, HEP User Facility. Fermilab is managed by Fermi Research Alliance, LLC (FRA), acting under Contract No. DE-AC02-07CH11359. 


\section{Overview}

- CMS data management needs and situation

- Review process and decision to adopt Rucio

- CMS Rucio infrastructure

- Plans for transition

- Current status and scale tests

- [User data management] 


\section{Current CMS Data Management}

- Data on tape O(100 PB) and disk O(50 PB)

- 8 sites with tape, $\mathrm{O}(100)$ with managed disk

- Production file size $\mathrm{O}(1 \mathrm{~GB})$, user file size $\mathrm{O}(100 \mathrm{MB})$

- Per day transfers 2 PB, 1 M files (user \& production)

- Similar scale for next 6-7 years, increases 50x in 2026

- Current data management is done by PhEDEx

- Each site hosts a PhEDEx agent to manage its own data including tape

- Requires non-trivial effort at each of our sites

- Maintains a database of the desired state (files at sites); issues FTS commands to achieve it

- PhEDEx is aging and we realize its lifetime is limited

- A higher layer, Dynamo makes PhEDEx requests to dynamically distribute and clean up data

- Separate physics meta-data catalog (DBS) 


\section{CMS Selection Process}

- Performed an evaluation of Rucio from early 2018 through summer 2018 - July 2018 - CMS conducted a down-select review at which Rucio was chosen

- Cited an existing product with a development plan for the future

- Obviously collaboration within LHC/HEP was a big plus

- Improve the long term sustainability of our tools

- Began a methodical transition to Rucio in Fall 2018

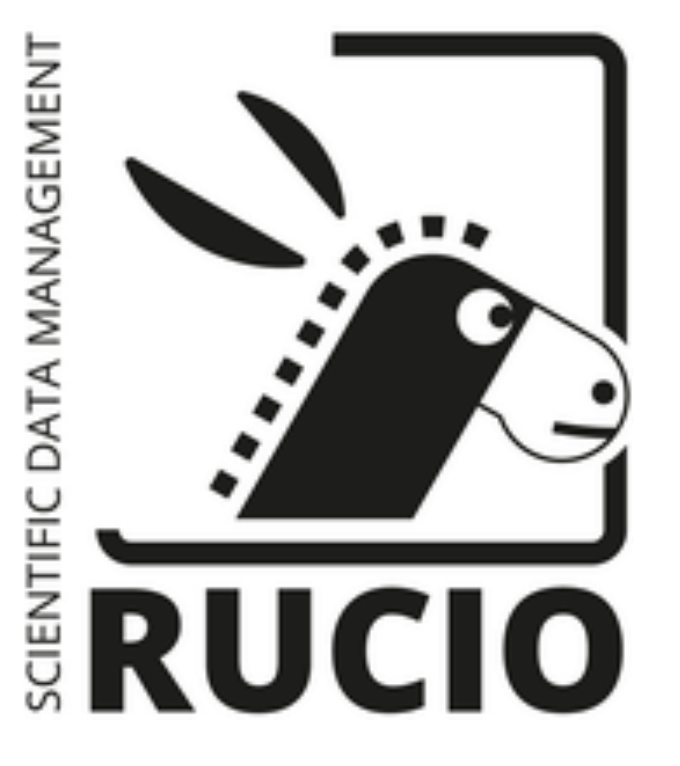




\section{CMS vs. Rucio Data models}

- CMS data stored in a three tiered structure:

- Files - target size 4 GB

- Blocks - usually about 500 files, designed to be a unit that can be stored and transferred at one site

- Dataset - some number of blocks, has a physics meaning (usually complete at a site)

- All many:one maps, not many:many (like rucio)

- Not perfect but fits OK into Rucio model:

- CMS Block $\rightarrow$ Rucio Dataset

- CMS Dataset $\rightarrow$ Rucio Container

- CMS has a single namespace of data with data types organized by directory

- Use a map of LFN (logical) to PFN (physical) namespaces

- We used Rucio's plugin and RSE attributes to implement this 


\section{CMS Rucio Infrastructure}

- Based on Docker, Kubernetes (k8s), Helm, OpenStack, Oracle

- Helm enables minimal config changes for CMS

- Zero to operating cluster is $\sim 30$ minutes

- Upgrades are nearly instantaneous

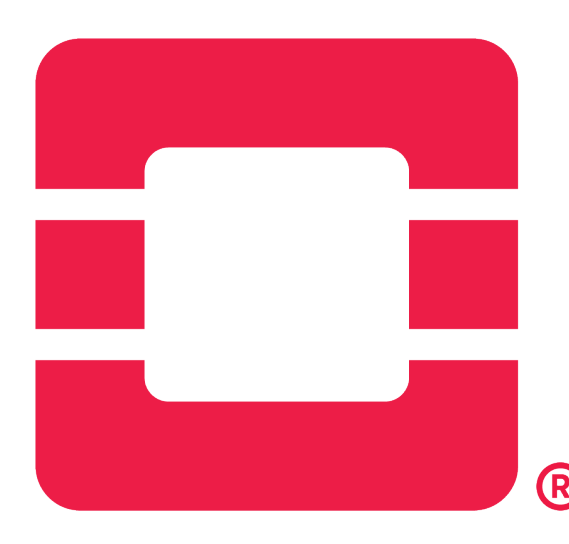

- All components of Rucio and supporting code are operating in k8s

- Monitoring, logging, proxy renewal, synchronization also in k8s Pods or CronJobs - Many 3rd party pieces built with helm as well

- ATLAS also moving towards $\mathrm{k} 8 \mathrm{~s} / \mathrm{helm}$ - collaborative effort

- Liveness checks give automatic restart

- Allows us to have production and testbed on a shared set of resources

- Developers' environment is smaller version of central cluster(s) 


\section{Transition: Syncing data between PhEDEx \& Rucio}

- Critical part of transition is moving all the metadata over

- Sync scripts keep replica info for a site in sync between PhEDEx and Rucio

- Define these Rucio endpoint mirrors as read-only (no transfers in, no deletions)

- Can be used as a source for additional replicas. During the transition period we will also have a [Site]_Test endpoint which writes to a test part of the physical namespace

- Currently uses REST APIs of Rucio and PhEDEx. May switch to direct database interface 


\section{Transition plan starting with NanoAOD}

- NanoAOD is the smallest CMS data tier: $\mathrm{O}(1 \mathrm{kB}) /$ event

- Goal: transition all management of NanoAOD to Rucio as a test case

- Good candidate; only read by end-users, easily reproduced

- Step 1: Sync all data on NanoAOD from PhEDEx to Rucio

- Step 2: Develop Rucio subscriptions and rules to distribute NanoAOD to test space

- Current size is 1M files/430 TB (250k/100 TB unique) in CMS

- Qualification test detailed on next slides

- Step 3: Publish NanoAOD directly into Rucio; use Rucio as the full data location store

- Rucio distributes NanoAOD with subscriptions and/or rules

- PhEDEx distributes all other Tiers of data

- Sync non-NanoAOD data from PhEDEx to Rucio.

- All CMS software will lookup data in Rucio

- Other data tiers can be migrated by repeating step 3 


\section{Million File Test}

- Make a total of 5 copies of NanoAOD

- 1 copy in Americas, Asia/Russia, and 1/2 of Europe. 2 copies in other 1/2 of Europe

- Regions were defined by bandwidth between sites

- Total stats replicated were $450 \mathrm{k}$ files $299 \mathrm{k}$ datasets. Total size $320 \mathrm{~TB}$

- Also tested a cleanup campaign of placed files

- Used Rucio subscriptions: Generate placement rules based on dataset metadata

- We had to re-tag all the relevant datasets as "New" to trigger the subscription

- Subscriptions are still generating rules as new datasets are added to Rucio by sync

- Workflow:

- Rucio daemon scans "new" datasets, creates rules

- Rule engine demands new replicas (minimal to satisfy rules)

- Submitter daemon makes transfer requests to FTS 


\section{Rule creation during and after test}

Transmogrifier new rules -

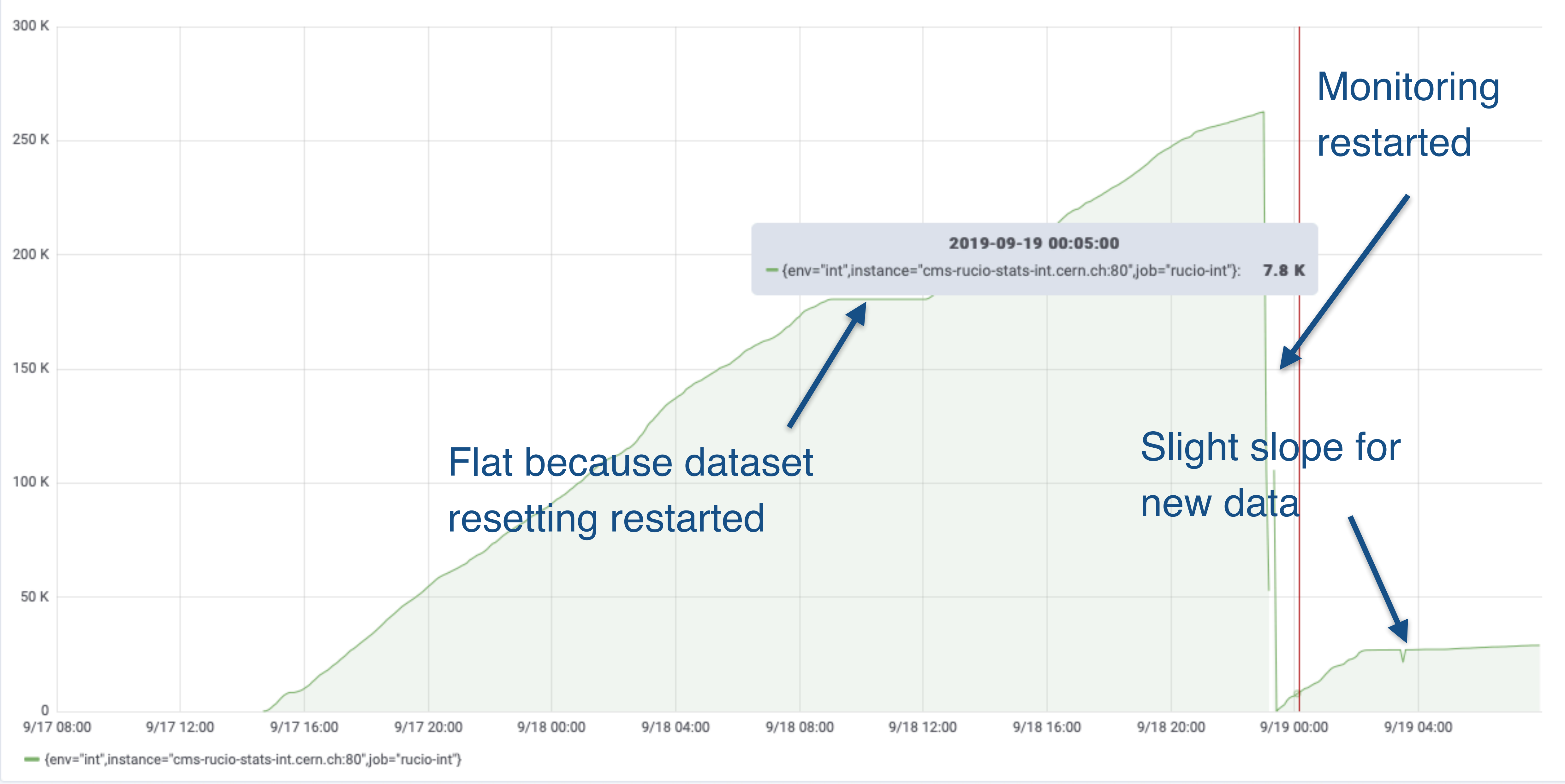




\section{Rucio throughput during test}

Million file

injection ends

Files

Transfered

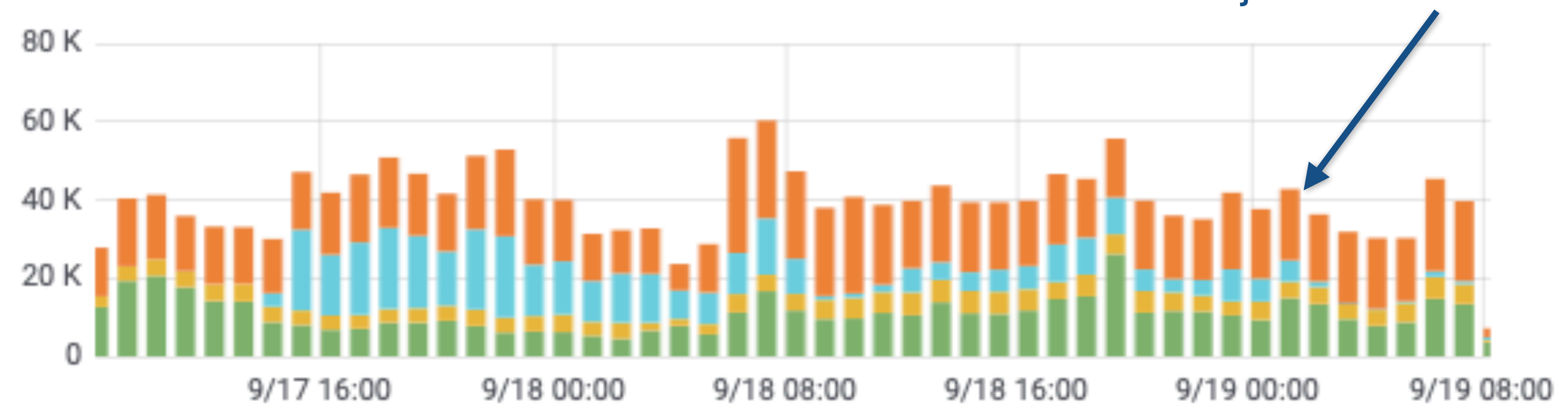

Bandwidth $100 \mathrm{~Gb} / \mathrm{s}$

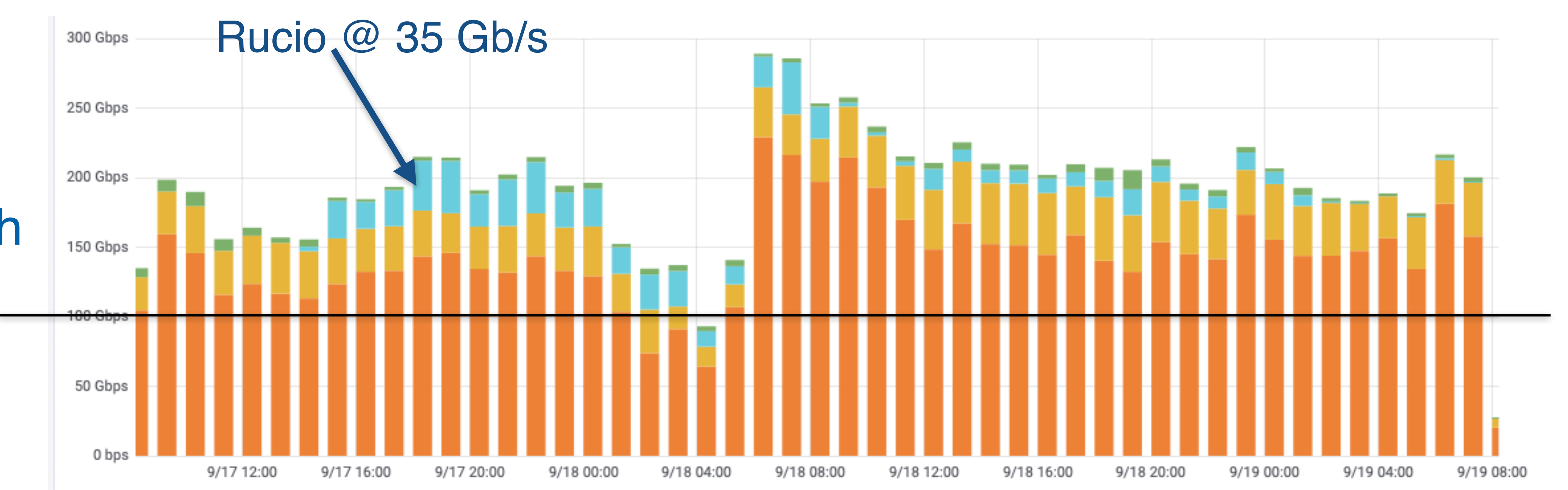

PhEDEx PhEDEx

Rucio

Users 


\section{Existing software needs to interface with Rucio}

- Orchestrator system for workflow management

- In the process of rewriting DM aspects of this for existing DM tools, adopting to Rucio is simple

- Input data placement is in pre-production testing

- Output data placement and data locking to be done

- Adapting workflow management system (WMAgent)

- A test agent which uses Rucio for data discovery is being tested

- Next step, development underway, is to publish data into Rucio

- Expect first tests this year

- Data aggregation service can already use Rucio as a source 


\section{Current CMS User Data Management}

- No user data management; movement and metadata only

- All done with AsyncStageOut (ASO) which is a thin layer on top of FTS

- Produced at Site A, moved to Site B (user has a relationship with Site B)

- User has a storage quota at Site B as part of site pledge to CMS

- Can never be moved to Site $C$ and have that reflected in DBS

- Some user data is completely unmanaged

- Rucio will give us new capabilities for user data handling 


\section{User Data Management with Rucio}

- First version of this is now available

- Users will be given quota at a geographically associated site

- Make dataset rule (for final state stageout) on task submission

- Register output files and attach to dataset on job completion

- Final data owned by CMS, initially data staged to temp area owned by user

- Job runs with user credentials

- Solution: Temporary endpoint is "non-deterministic" which maintains PFN explicitly

- Roll out for user data management is orthogonal with production.

- Bar to replace ASO is lower. 


\section{Conclusions}

- Rucio is a good match for CMS data management needs

- Transitioning an operating experiment at this scale is a non-trivial process

- CMS has tested Rucio near the LHC Run 3 scale needed

- Expect significant benefits from shared code base and operational experience with ATLAS and others - Operational Intelligence paper in Track 3

- The final transition will occur in 2020

- We expect to collaborate on future development which enable Rucio to function in the HL-LHC era 